\title{
BMJ Open Sonographic Evaluation of the Mechanism of Active Labor (SonoLabor Study): observational study protocol regarding the implementation of the sonopartogram
}

To cite: Dira LM, Tudorache S, Antsaklis P, et al. Sonographic Evaluation of the Mechanism of Active Labor (SonoLabor Study): observational study protocol regarding the implementation of the sonopartogram. BMJ Open 2021;11:e047188. doi:10.1136/ bmjopen-2020-047188

- Prepublication history for this paper is available online. To view these files, please visit the journal online (http://dx.doi. org/10.1136/bmjopen-2020047188).

The SonoLabor Study recruited patients during JanuaryDecember 2016 for a pilot study of 168 deliveries. Data analysis was completed in January 2017 and the results were communicated at the 27th World Congress on Ultrasound in Obstetrics and Gynecology. Then the project was discontinued, due to the lack of resources. Given the birth flow in the centres involved, the sample size required, the acceptability previously recorded and the disponible sonographers for intrapartum US evaluations, we should expect that the study should be completed during a 2-year period of time.

Received 22 November 2020 Accepted 23 June 2021

Check for updates

(C) Author(s) (or their employer(s)) 2021. Re-use permitted under CC BY-NC. No commercial re-use. See rights and permissions. Published by BMJ.

For numbered affiliations see end of article.

Correspondence to

Dr Monica Laura Cara;

monica-laura.cara@outlook.com

\author{
Laurentiu Mihai Dira (D) , ${ }^{1}$ Stefania Tudorache, ${ }^{1}$ Panagiotis Antsaklis, ${ }^{2}$ \\ George Daskalakis (D) , ${ }^{2}$ Dagklis Themistoklis, ${ }^{3}$ Smaranda Belciug, ${ }^{4}$ \\ Ruxandra Stoean, ${ }^{4}$ Marius Novac, ${ }^{5}$ Monica Laura Cara (i) , ${ }^{6}$ Roxana Dragusin, ${ }^{1}$ \\ Maria Florea, ${ }^{1}$ Ciprian Patru, ${ }^{1}$ Lucian Zorila, ${ }^{1}$ Rodica Nagy, ${ }^{1}$ Dan Ruican, ${ }^{1}$ \\ Dominic Gabriel Iliescu ${ }^{1}$
}

\section{ABSTRACT}

Introduction Over the last decades, a large body of literature has shown that intrapartum clinical digital pelvic estimations of fetal head position, station and progression in the pelvic canal are less accurate, compared with ultrasound (US) scan. Given the increasing evidence regarding the advantages of using US to evaluate the mechanism of labour, our study protocol aims to develop sonopartograms for fetal cephalic presentations. They will allow for a more objective evaluation of labour progression than the traditional labour monitoring, which could enable more rapid decisions regarding the mode of delivery.

Methods/analysis This is a prospective observational study performed in three university hospitals, with an unselected population of women admitted in labour at term. Both clinical and US evaluations will be performed assessing fetal head position, descent and rotation. Specific US parameters regarding fetal head position, progression and rotation will be recorded to develop nomograms in a similar way that partograms were developed. The primary outcome is to develop nomograms for the longitudinal US assessment of labour in unselected nulliparous and multiparous women with fetal cephalic presentation. The secondary aims are to assess the sonopartogram differences in occiput anterior and posterior deliveries, to compare the labour trend from our research with the classic and other recent partogram models and to investigate the capability of the US labour monitoring to predict the outcome of spontaneous vaginal delivery.

Ethics and dissemination All protocols and the informed consent form comply with the Ministry of Health and the professional society ethics guidelines. University ethics committees approved the study protocol. The trial results will be published in peerreviewed journals and at the conference presentations. The study will be implemented and reported in line with the Strengthening the Reporting of Observational Studies in Epidemiology statement.

Trial registration number ClinicalTrials.gov Registry (NCT02326077).
Strengths and limitations of this study

- The multicentre design on representative population and the blinded clinical/ultrasound assessment aim to intercept the potential sources of bias.

- The SonoLabor Study differs from previous studies as it aims to assess all stages of labour, rather than just the second stage of labour to elaborate nomograms for the longitudinal ultrasound assessment of labour in unselected lowrisk population, an important issue of the future sonopartograms.

- Sonographic and clinical evaluation of the labour progression in any cephalic presentation (not only with occiput anterior position).

- Our study aims to develop curves for labour monitoring which will be not only objective, but also adapted to contemporary practice. Clinical studies showed that the pattern of labour progression and the present characteristics of the partogram differ significantly from the traditional Friedman curve.

- The high number of labouring women needed to investigate the characteristics of each clinical situation targeted in the study design. In order to produce specific sonopartograms regarding the maternal characteristics and occiput position, an important number of patients would be required, that may not achievable during our present research. We hope that the publication of our study protocol, dissemination of the results and the storage of the anonymised collected data in a research depository will serve to future larger studies that will help to collect or complete the necessary data.

- The concept of normality is population based and depends on various management attitudes (for example, epidural analgesia, active management of labour), different characteristics of the partogram are observed that may affect generalisability. 


\section{INTRODUCTION}

Over the last decades, a large body of literature has shown that clinical digital pelvic estimations of fetal head position, station and progression in the pelvic canal are not accurate during the first ${ }^{1}$ and second stage of labor, ${ }^{1-6}$ poorly reproducible when compared with ultrasound (US), ${ }^{7-9}$ poorly reliable $^{10-12}$ experience dependent ${ }^{14}$ and often inexact in challenging labour circumstances, such as: prolonged first stage of labour, ${ }^{13}$ cases with arrested cervical dilation, ${ }^{14}$ obstructed labour, ${ }^{15}$ fetal head engagement, ${ }^{10}{ }^{16}$ posterior and transverse occiput locations, ${ }^{614}$ or caput. ${ }^{4}$ This may imply significant consequences on the decision of the appropriate delivery mode, because digital examination is less reliable especially when obstetrical interventions are more likely to be needed. ${ }^{15}$ 17-21 Intrapartum sonographic evaluation may not provide a solution for all these conditions mentioned above, but previous studies have demonstrated the potential to decrease the rate of late caesarean extractions in prolonged labour cases, and the various approaches proposed in the literature were considered by our study design. Many studies provided sonographic data regarding the fetal head descent/ progression (FHPr) in the second stage of labour and proposed several easily measurable and reliable parameters, capable to predict the vaginal or operative outcome of the delivery with occiput anterior positions. ${ }^{22-28}$ The literature regarding US evaluation in the first stage of labour is less, but based on available data US evaluation appears to be useful for the prognosis of labour. ${ }^{29-31}$

Given the increasing evidence regarding the advantages offered by using US in labour, our group concluded that the development of a sonopartogram, as an adjuvant to or a replacement of traditional labour monitoring, provides the setting for a more objective evaluation of labour progression, which would enable more rapid decisions regarding the mode of delivery. ${ }^{32} 33$ Intrapartum US evaluation is not meant to change the standard principles for labour mechanism evaluation, but to provide accurate evaluation of the main parameters involved: fetal head position and rotation, fetal head progression and engagement.

The SonoLabor Study aims to provide new objective evidence regarding the evaluation of the mechanism of labour with US. There is little information in the literature regarding the ultrasonographic monitoring of the entire active labour. ${ }^{34-36} \mathrm{~A}$ recent proof-of-concept study showed that the sonopartogram is feasible in most cases. ${ }^{34}$ However, a study of the paired clinical and sonographic assessments of labour in a large, unselected population has not yet been conducted. Furthermore, there are no nomograms for the US monitoring of labour. Nowadays, the use of US in labour is generally limited to research settings and a relatively small number of women have been studied. Therefore, efforts should be made to describe the value of an objective partogram in general practice.

This study is designed to produce an original multicentre longitudinal assessment of the mechanism of active labour, including both stages, in a representative population, using concomitant blinded clinical and sonographic evaluations in unselected low-risk parturient women at term. A unique point of our protocol is the comparative evaluation of the US parameters for various clinical situations. This may facilitate the use of different nomograms in labour, adapted to the clinical characteristics of the labouring woman. Another strength of our study is the multicentre design that is useful to achieve a proper study size and an opportunity to compare the data recorded in different settings.

The aim of this paper is to describe the protocol of the study.

\section{Objectives}

The primary objective of this study is the development of nomograms for US-measured variables during labour in unselected nulliparous and multiparous women at term with fetal cephalic presentation.

The secondary objectives of the study are the following:

- To compare the US pattern of labour evolution in nulliparous and multiparous women.

- To study the influence of occiput position, body mass index (BMI), parturient age on the labour progression evaluated by US.

- To correlate the labour trend from our study with the Friedman studies ${ }^{37} 38$ and other recent research on the partogram ${ }^{39}$ regarding the progression of labour by means of objective US evaluation.

- To correlate the US and standard clinical findings regarding the mechanism of labour, for example, fetal occiput position and head descent during active labour.

- To investigate the correlations between the data of the participating centres.

- To analyse the temporal variation of the sonographic measurements in spontaneous vaginal delivery versus obstructed labour in nulliparae versus multiparae.

- To analyse the evolution of the sonographic measurements in spontaneous vaginal delivery versus obstructed labour in fetuses with occiput anterior versus those with persistent occiput posterior.

- To investigate the value of combined US measurements to predict the outcome of vaginal delivery.

\section{METHODS AND ANALYSIS}

\section{Study design and setting}

This is an observational cohort prospective study, which will take place in three tertiary maternity hospitals (University Emergency County Hospital Craiova, Alexandra University Hospital of Athens and Ippokrateion Hospital Thessaloniki), with more than 4000 deliveries per annum. The study aims to record simultaneously the labour progress by clinical and US evaluations in women in labour at term, with singleton cephalic presentation. We will include low-risk pregnancies, according to the criteria defined in the Participants section.

\section{Patient and public involvement}

We conducted a previous study ${ }^{40}$ during the development of this research question, where we evaluated the acceptability of the method and found that the vast majority of 
labouring women $(98 \%)$ agree with the supplementary US investigation protocol and the demographic characteristics did not influence the rate of acceptance. Most of the women (93\% of accepters and $75 \%$ of decliners) had little difficulty deciding whether or not to have the scan protocol. All women who were scanned during labour found it an acceptable experience, and only $21 \%$ of women without epidural anaesthesia rated the perceived difficulty as 'mild' or 'discomforting'. Women rated having the intrapartum scan as being significantly less difficult than having a cervical smear, transvaginal scan or having a digital clinical evaluation. Two-thirds $(67 \%)$ of the patients expressed increased confidence while being able to follow along the medical personnel the FHPr on the US screen. Almost all of the consenting women $(97 \%)$ who had the intrapartum US scans and all the four decliners said they would definitely or probably agree such US monitoring in a future labour, if this technique is proven useful for the labour outcome.

\section{Participants}

All pregnant women admitted in active labour at term are considered eligible for the study. They will be consecutively included in the study, depending on the availability of the US operators involved in the study. We will try to attract a large team of collaborators, in order to investigate as many eligible cases as possible. Cases planned for elective caesarean section, or involving imminent intention to deliver, with non-cephalic presentation, intrauterine death, multiple pregnancies or resulting neonates weighing less than $2500 \mathrm{~g}$ or more than 4000 $\mathrm{g}$ will be excluded from the study. Also, we will exclude women with previous cervical surgery (eg, cone biopsy, cervical cerclage), those younger than 18 years, or those considered in the opinion of the researcher as having language or learning impairment.

Following the clinical evaluation, women will be admitted in the first stage of labour when there are regular painful contractions and there is a progressive cervical dilatation from $4 \mathrm{~cm}$, and the second stage will be established based on the finding of full dilatation of the cervix $^{41}$ regardless of whether the parturients underwent artificial rupture of the membranes, oxytocin augmentation or epidural anaesthesia.

Gestational age will be determined by the last menstrual period in women with regular menses, confirmed with US dating, preferably during the first trimester. If the first trimester biometry is not consistent with the menstrual dating by more than 1 week, gestational age will be established based only on the sonographic evaluation. In women with irregular menses, the gestational age will be determined solely based on the first fetal biometry evaluation in in the first half of pregnancy.

\section{Procedures}

Recruitment

During their usual consultation in the labour ward, in an eligible case, the physician on duty provides brief information about the research and invites the patient to take part in the study. If the patient shows interest in the study and meets the inclusion criteria, a face-toface appointment with the US operator is arranged. The details of the study and the potential benefits of the research will be thoroughly explained to the patient. The only direct benefit of the labouring women who participate in the study would be the communication between the obstetrician and sonographer regarding fetal head position (FHPo) when instrumental delivery is attempted, as presented in the Interventions section. If the patient agrees to participate in the study, written informed consent will be obtained.

\section{Interventions}

All pregnant women who meet the inclusion criteria will be assessed clinically by the physician on duty. The managing clinician is a senior consultant not involved in the study.

Clinical examinations will take place in women in active labour just before the US assessments (figure 1). The clinician will note the observations on a specially designed partogram-like sheet that will be used for women in labour who agree to participate in the study. The following labour parameters must be noted before US assessment:

- Cervical dilation in centimetres.

- FHPo-the evaluation of occiput position in both labour stages. Occiput position will be classified as occiput anterior (OA), occiput posterior (OP), left or right occiput transverse, left or right occiput anterior, or left or right occiput posterior. ${ }^{542}$

- FHPr-determined by the evaluation of head station in relation to the ischial spines.

- Presence of caput, with the approximate diameter.

- Presence of moulding and grading: closure of sutures with no overlap (grade 1), reducible bones overlap (grade 2) and irreducible overlap (grade 3).

Clinical examination will be followed by transabdominal and transperineal US evaluations conducted by obstetricians with appropriate training in US in labour, with minimum 1 year of experience in the field. Mobile and compact US machines will be used: Logic e (GE Healthcare, China), GE Voluson P6, Samsung R7, BenQ T3300 and ALOKA f31 equipped with 2-5 and 2-6 MHz 2D convex transducers.

The objectives of US evaluations are similar to those of standard clinical assessment. The purpose of US evaluations is to document the progression of labour using objective measurements for the main parameters involved in the mechanism of labour:

- FHPo, by determining occiput position.

- Fetal head rotation in the second stage of labour, by measuring the midline angle.

- FHPr, by evaluating the relation between the fetal head and maternal landmarks, using specific measurements: progression angle (PA), progression distance 


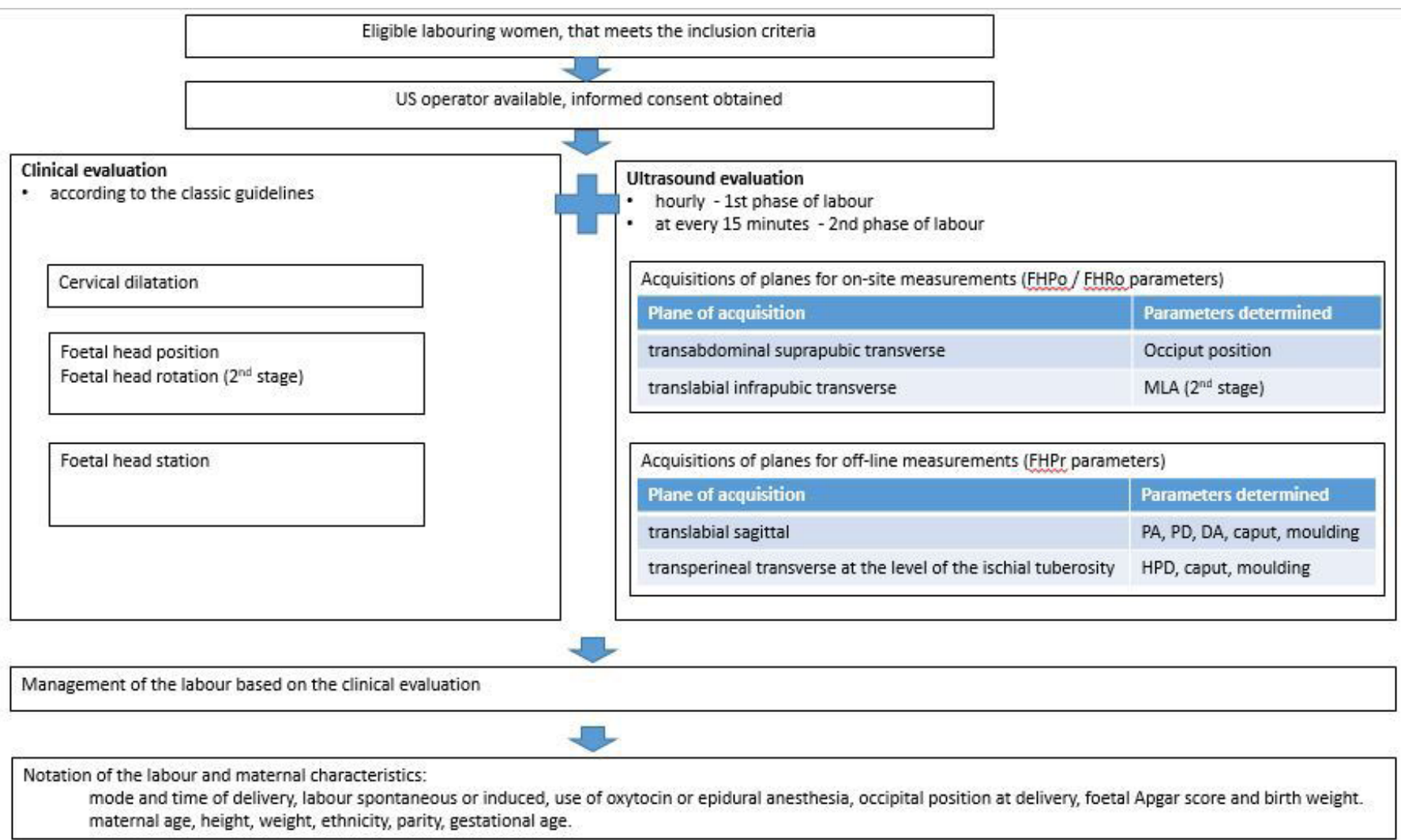

Figure 1 Implementation of the SonoLabor Study. DA, direction angle; FHPo, fetal head position; FHPr, fetal head progression; FHRo, fetal head rotation; HPD, head to perineum distance; MLA, midline angle; PA, progression angle; PD, progression distance; US, ultrasound.

(PD), head direction angle (DA) and head to perineum distance (HPD).

- Caput measurement, if present.

- Moulding notation, if present.

In table 1, we present the sonographic measurements, in relation to the acquisition planes and the features of labour mechanism that are involved. The images will be stored on the hard disk drive of the system for offline analysis and the measurements will be performed by the sonographer who evaluated the case, according to the techniques described in the literature ${ }^{522-274243}$ (table 1).

Because of ethical issues, the design of the study states that the attending obstetrician should be informed in case of clinical and US discordance when instrumental or operative delivery is attempted. The digital evaluation is considered to be correct if the FHPo is within $\pm 45^{\circ}$ of the US determination.

Cervical dilation will be evaluated only clinically, as the evaluation of this parameter is best achieved with digital assessment. $^{31}$

Timing: clinical and US scans is performed hourly until complete dilation (first phase of active labour) and at every $15 \mathrm{~min}$ after complete dilation (second phase). The purpose of the apparently high number of examinations was to obtain accurate information in each labour in terms of correlation of FHPr, FHPo and cervical dilatation. In a previous study in our clinic, this methodology proved to be acceptable for the parturients. ${ }^{40}$ The frequent evaluations in the second stage are meant to offer a better analysis of this critical stage of labour. Notation of time delivery will be used to calculate the time interval from each scan to delivery.

US images will be saved and stored on US hard disk during labour, then transferred by the sonographer to a designated personal computer storage unit after birth. The images will be reviewed during the following week, by the same sonographer, who will also input the offline measurement results into the database.

The sonographer and the clinician are blinded to each other's findings (except FHPo, during the circumstances mentioned above) as the specific measurements are performed afterwards, offline and labour management is conducted by the labour and delivery department personnel. During labour, the available sonographer cannot be completely blinded to clinical findings, as he/she will perform the scans at certain time intervals, depending on the labour stage, that is established by the clinician's cervical dilatation assessment. However, the sonographer will only record the images. The clinician will note the observations on a partogram-like sheet that is not available for the sonographer, who in turn, will perform the measurements offline, after birth.

Labour characteristics will be recorded by the clinician on the study datasheet: mode and time of delivery, neonatal Apgar score and birth weight, whether labour was spontaneous or induced, use of oxytocin or epidural 
Table 1 Acquisition of US planes, and US measurements performed offline, according to previous literature

\begin{tabular}{ll}
\hline Labour mechanism feature & Acquisition plane \\
\hline $\begin{array}{l}\text { Fetal head position (FHPo) in the } \\
\text { first stage of labour }\end{array}$ & $\begin{array}{l}\text { Transabdominal suprapubic } \\
\text { transverse plane }\end{array}$ \\
& \\
$\begin{array}{l}\text { FHPo in the second stage } \\
\text { of labour, evaluation of head } \\
\text { rotation }\end{array}$ & $\begin{array}{l}\text { Transperineal infrapubic transverse } \\
\text { plane (figure 3), with visualisation } \\
\text { of the cerebral midline }\end{array}$ \\
\end{tabular}

\section{US measurements}

Occiput position (figure 2)

Both clinical and US findings of the FHPo are recorded on a data sheet depicting a circle, like a clock, divided into 24 sections, each of $15^{\circ}$, and the position of the occiput is assigned as anterior (OA), posterior (OP), left anterior (LOA), right anterior (ROA), left posterior (LOP), right posterior (ROP), right transverse, left transverse. ${ }^{542}$

The position of the occiput is determined based on the identification of the midline, thalami, choroid plexus, cerebellum, orbits or occiput.

\section{- Occiput position}

- Midline angle (MLA) (figure 3)

The position of the occiput (OA, OP, LOA, ROA, LOP, ROP) is determined in a similar fashion transperineally in the 2nd stage of labour.

MLA is calculated ${ }^{25}$ based on the visualisation of the cerebral midline in relation to the anteroposterior axis of the maternal pelvis-rotation angle or MLA.

\begin{tabular}{|c|c|c|}
\hline \multirow[t]{2}{*}{ Fetal head descent/progression } & $\begin{array}{l}\text { Transperineal translabial sagittal } \\
\text { plane }\end{array}$ & $\begin{array}{l}\text { Progression angle } 2627 \text { (figure 4), as the angle between the } \\
\text { longitudinal axis of the pubic symphysis and the line running } \\
\text { from the anterior edge of the pubic symphysis tangentially } \\
\text { to the leading edge of the fetal skull. } \\
\text { Progression distance } \\
\text { between a vertical line from inferior apex of the symphysis } \\
\text { (infrapubic line) and the leading edge of the fetal skull. } \\
\text { Head direction angle }{ }^{23} 24 \text { (figure } 4 \text { ), as the direction of the } \\
\text { line perpendicular to the widest diameter of the fetal head, } \\
\text { with respect to the infrapubic line. }\end{array}$ \\
\hline & $\begin{array}{l}\text { Transperineal translabial transverse } \\
\text { plane, at the level of the ischial } \\
\text { tuberosity, applying firm pressure } \\
\text { without creating discomfort, and } \\
\text { the transducer moved and angled } \\
\text { until the shortest distance to the } \\
\text { fetal skull is visualised }\end{array}$ & $\begin{array}{l}\text { Head to perineum distance, }{ }^{43} \text { as the shortest distance from } \\
\text { the outer bony limit of the fetal skull to the skin surface of } \\
\text { the perineum (figure } 4 \text { ). }\end{array}$ \\
\hline
\end{tabular}

US, ultrasound.

anaesthesia, occipital position at delivery. Maternal characteristics will be retrieved from the hospital records (patient files) by the personnel involved in data centralisation: age, height, weight, ethnicity, parity, gestational age.

The US labour assessments should not be biased by confounders that influence the quality of the clinical evaluations in labour (obesity, anterior placenta, caput, moulding), because the visualisation of the fetal skull and pubic symphysis is easily achievable even in such conditions. To ensure protocol fidelity, all sonographers will have completed a 1-day workshop and will participate in group supervision sessions programmed weekly in the first month of the study. This approach proved to be successful during the previous pilot study conducted in our centre.
The information provided to the clinician regarding the US determination of the FHPo in case of clinicalsonographic discordance before instrumental or operative delivery could represent a theoretical bias of the study. However, many tertiary centres already use the US determination of the FHPo in such situations, and this aspect does not interfere with the objectives of our study.

\section{Outcome measures}

Primary outcome

The primary objective of this study is the elaboration of nomograms for the longitudinal US assessment of labour in unselected nulliparous and multiparous women with fetal cephalic presentation. The nomograms represent the evolution of the progression markers (PA, DA, PD, $\mathrm{HPD})$ in relation to time and cervical dilatation. 

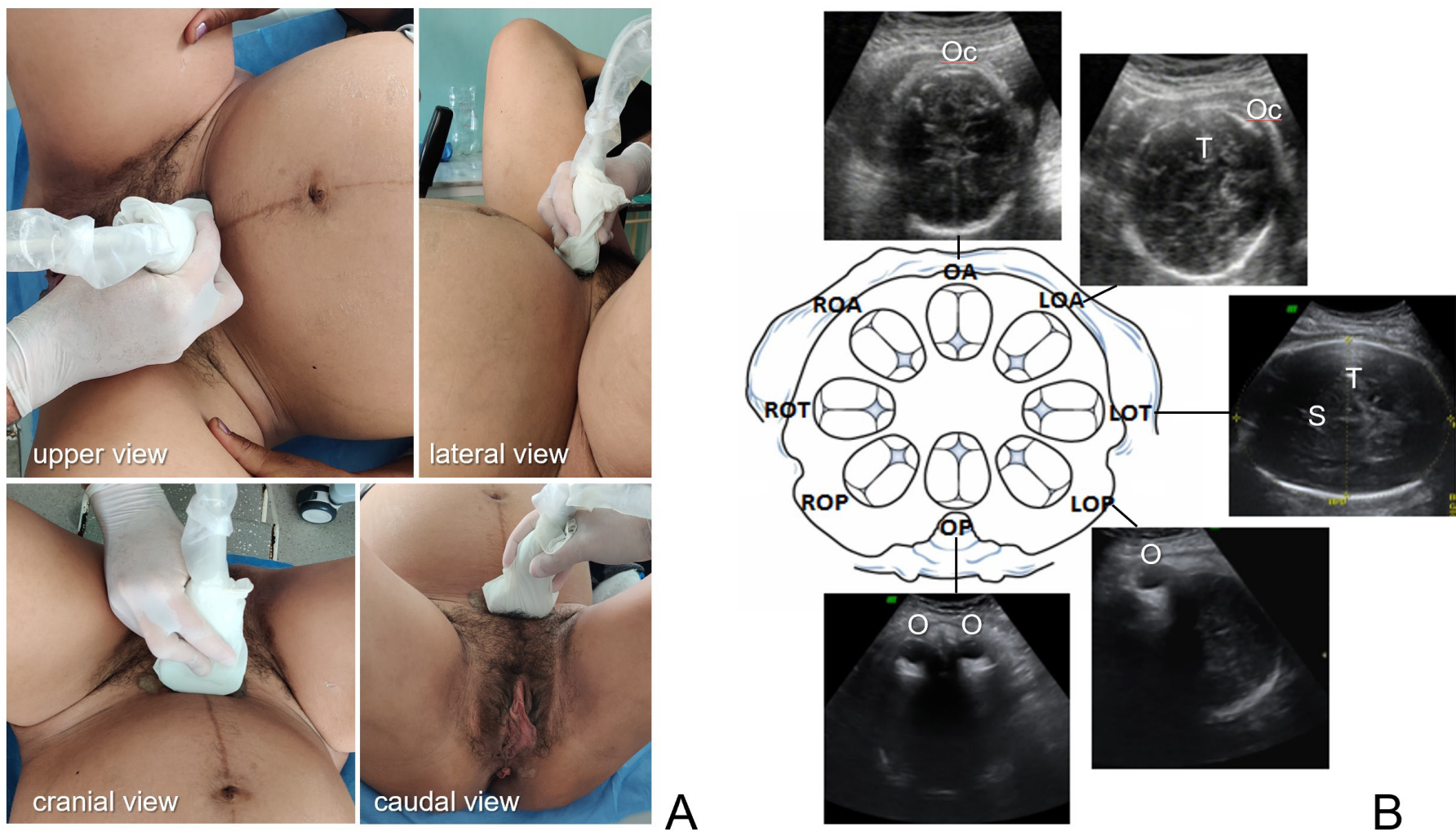

Figure 2 Ultrasound determination of the occiput position in the first stage of labour. (A) Example of the probe orientation in the transabdominal suprapubic transverse plane. (B) FHPo determination in the plane described in (A), based on the identification of cranium or cerebral structures: occiput (Oc), thalamus (T), interhemispheric septum (S), orbits (O). FHPo, fetal head position; LOA left occiput anterior; LOP, left occiput posterior; LOT, left occiput transverse; OA, occiput anterior; OP, occiput posterior, ROA right occiput anterior; ROP, right occiput posterior; ROT, right occiput transverse.

\section{Secondary outcomes}

- To compare the US pattern of labour evolution in nulliparous and multiparous women.

- To study the influence of occiput position, BMI and parturient age on the mechanism of delivery evaluated by US.

- To compare the labour clinical trend from our study data with the Friedman studies ${ }^{35} 36$ and other recent research on the partogram. ${ }^{39}$

- To correlate the US findings with classic clinical estimations:

- Correlation of the FHPo determined by US with FHPo clinically estimated (by digital vaginal evaluation (VE)).

- Correlations between the US FHPr parameters and between US and clinical (head station) FHPr parameters.

- The concordance between the fetal head station evaluations derived from US measurements and clinical digital estimations.

- To investigate the correlations between the data of the participating centres.

- To analyse the evolution of the sonographic measurements in spontaneous vaginal delivery versus obstructed labour cases, in nulliparae versus multiparae, and in occiput anterior deliveries versus those with persistent occiput posterior.

- To evaluate the capability of the US technique to predict the labour outcome (vaginal or caesarean birth) in both nulliparous and multiparous women.

\section{Data collection and management, and quality control}

To ensure protocol fidelity, the sonographers will have completed a 1-day workshop and will participate in group supervision sessions, programmed weekly in the first month of the study. This approach proved to be successful during the previous pilot study conducted in our centre. The following procedures are to be followed:

- At the initial workshop, the standardised procedures regarding data collection, encoding of the clinical and US data and electronic storage will be established.

- The principal investigators will organise training sessions to provide instructions on the protocol and study procedures for the sonographers at the beginning of the study.

- Monthly meetings will take place between study site personnel to discuss issues related to the conduct of the study and supplementary convocations will be announced whenever necessary. 

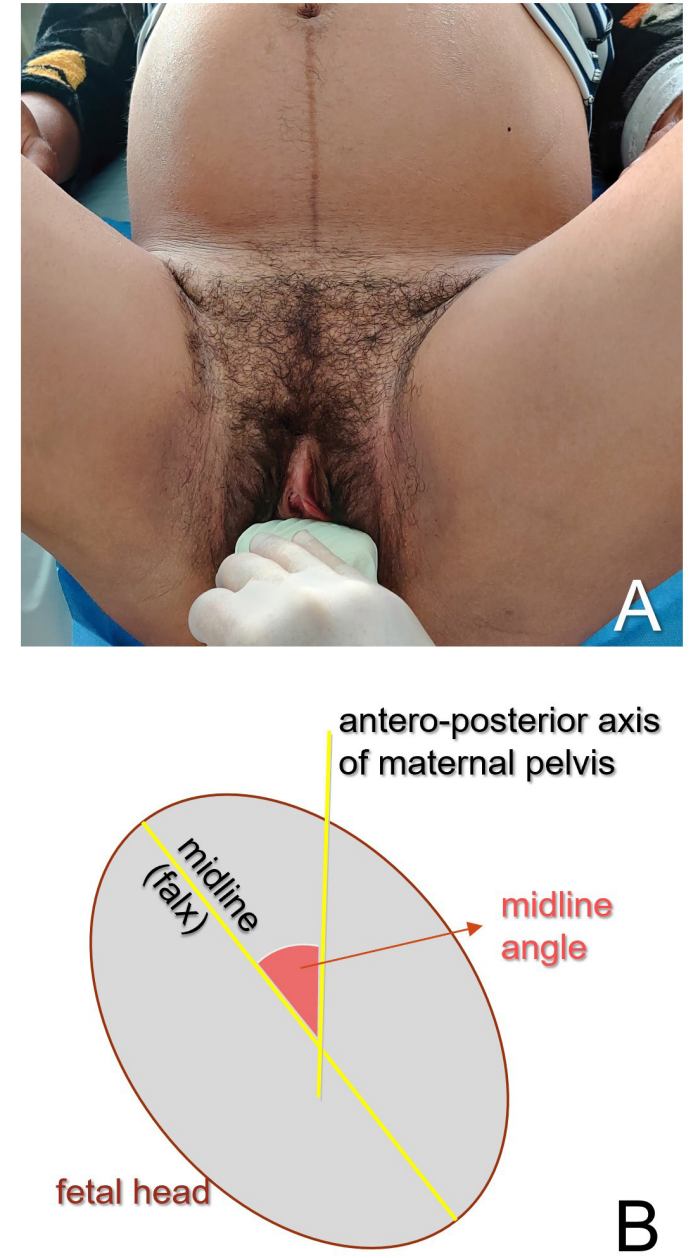
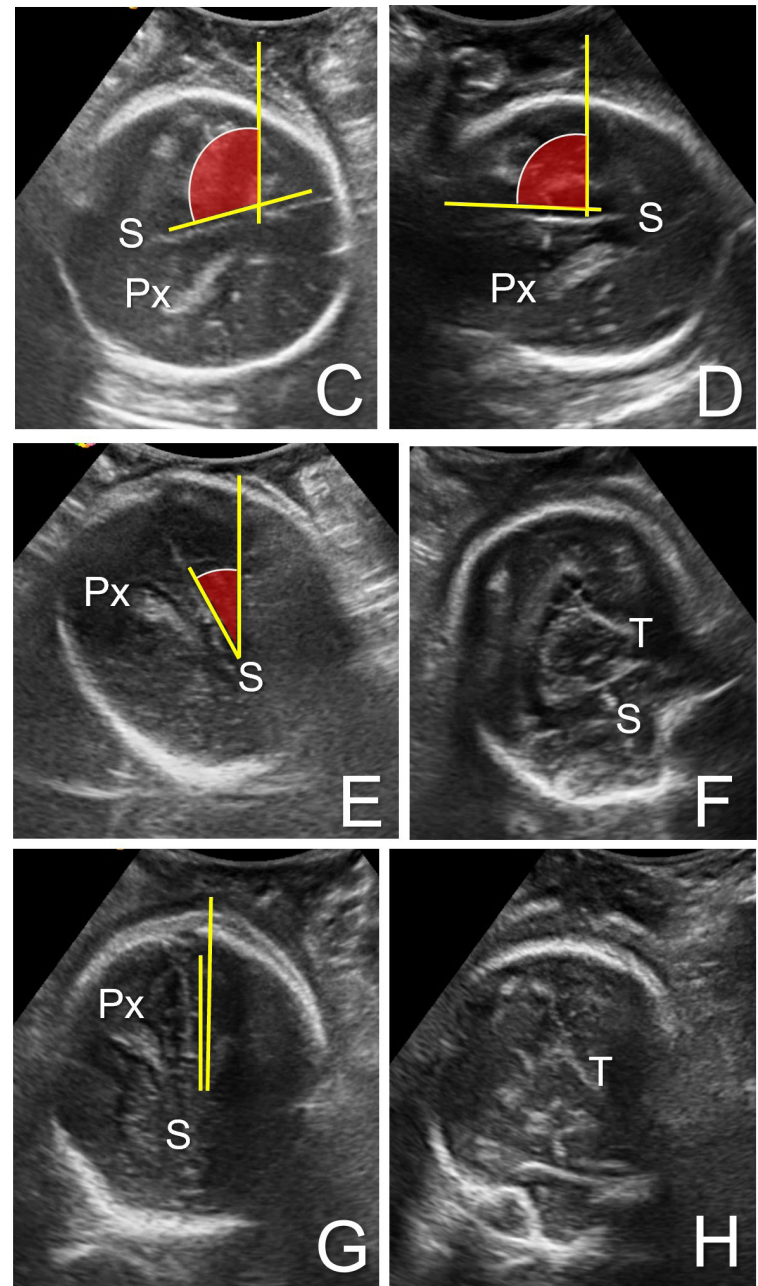

Figure 3 Ultrasound determination of the occiput position in the second stage of labour. (A) Example of the probe orientation in the transperineal infrapubic transverse plane. (B) Schematisation of the structures visualised and the measurement of the midline angle between the midline (falx cerebri) and the anteroposterior axis of the maternal pelvis. $(\mathrm{C}-\mathrm{H})$ Presentation of an example with midline angle measurement and evolution during anterior rotation of a transverse occiput. Occiput position is identified based on the visualisation of the cerebral midline (interhemispheric septum, (S) and choroid plexus (Px) direction (divergent posteriorly), or thalami aspect (triangular, with the base anteriorly). Midline angle gradually decreases during the anterior occiput rotation from the transverse position $(C, D)$, as it reaches right anterior $(E, F)$ and anterior (infrapubic) $(G, H)$ positions.

- The principal investigators in the three centres will be available for consultation by telephone at request.

- Interim analyses monthly-the data manager will evaluate the data with the statistics personnel and will conduct a quality review of the database. The results of the interim analyses will be discussed between the principal investigators, who decide whether to continue, stop or modify the trial.

- All the collected data will be anonymised. The data will be collected by the research team, processed and stored in the www.zenodo.org research depository.

\section{Statistical methods}

\section{Sample size estimation}

Although several studies have investigated the clinical course of labour, until present we do not have data regarding the nomograms for US evolution of labour. The number of patients enrolled in the clinical partogram studies varies widely. However, the Friedman's study that still serves as the basis of how most physicians define normal labour enrolled 500 nulliparous women at term. ${ }^{37}{ }^{38}$ On the other hand, we have a recent large, but retrospective study that analysed the clinical labour records of more than 62000 women from 19 hospitals across the USA and concluded that these criteria created 50 years ago may no longer be applicable to contemporary obstetric populations and for current obstetric management. ${ }^{39}$

Regarding imaging studies, the prospective research on the trend of the labour progress using intrapartum transperineal US gathered less than 100 cases each. ${ }^{34} 3644$

The primary outcome of our study will be centile charts for each US progression marker in relation to time.

An important challenge of our study is to achieve a sufficient number of $\mathrm{OP}$ cases in both nulliparous and 


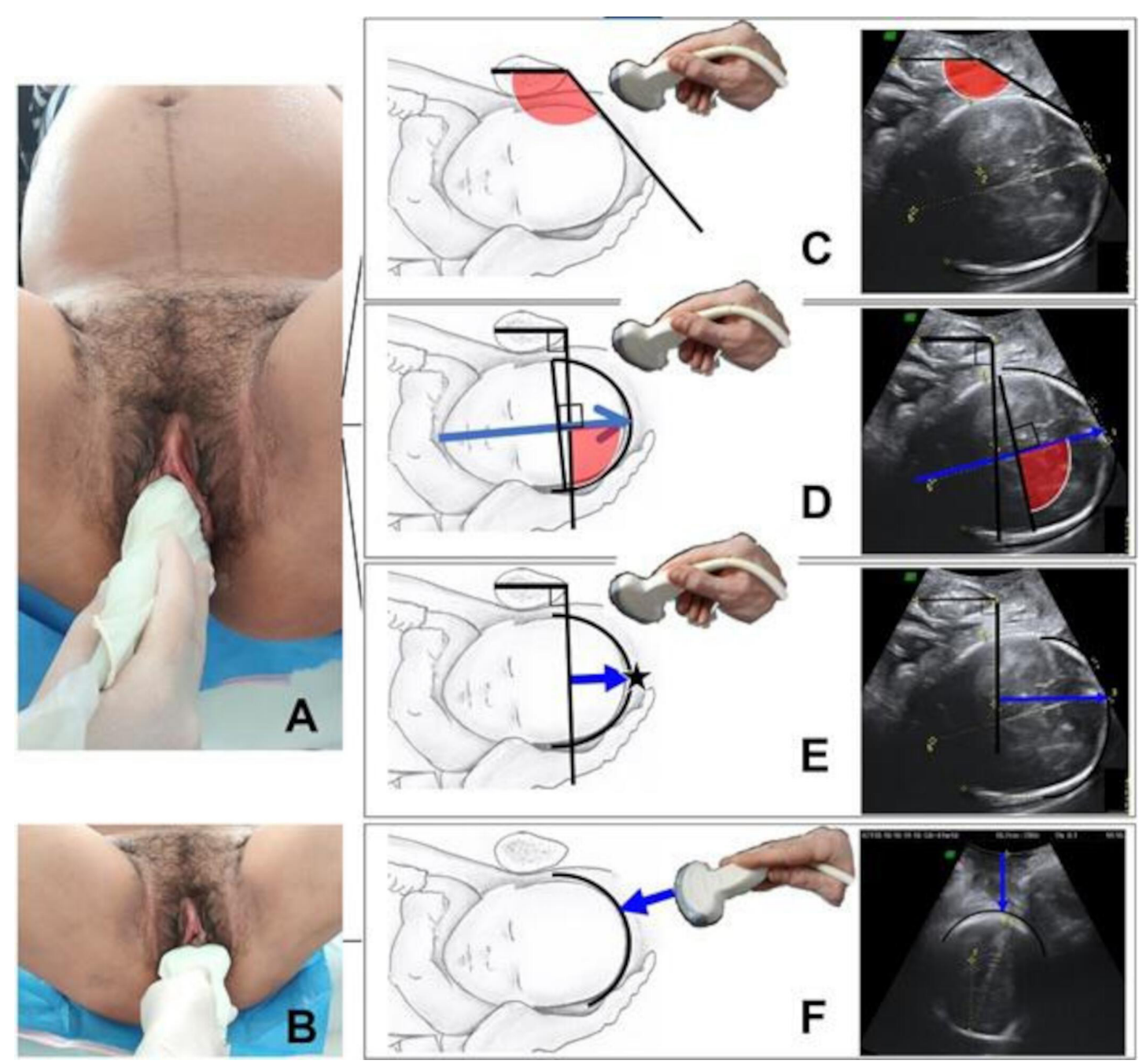

Figure 4 Ultrasound determination of the fetal head descent/progression. Placement of the transducer in the infrapubic translabial sagittal plane (A) and infrapubic transverse plane (B). (C) Measurement of the progression angle between the long axis of the pubic symphysis and a line extending from its most inferior portion tangentially to the fetal skull. (D) Measurement of the direction angle as the angle between the major longitudinal axis of the fetal head (perpendicular to the biparietal diameter) and the infrapubic line. (E) Measurement of the progression distance as the minimal distance between the infrapubic line and the leading part of the fetal skull (star). (F) Measurement of the head to perineum distance as the shortest distance from the skin surface of the perineum to the outer bony limit of the fetal skull.

multiparous women. According to the Central Limit Theorem and the Large Enough Sample Condition, a sample size of at least 30 items is sufficient for describing a 'normal' behaviour of the sample, even if it is not governed by the Gaussian distribution. By looking at the t-table, we can see that when using around $30 \mathrm{df}$, the value of $t$ becomes approximately equal to the value of the $z$ statistics. ${ }^{45}$ Taking into account previously published studies, the overall rate of occiput posterior deliveries in nulliparous is around $7.2 \%$, whereas for the multiparous deliveries is around $4 \%$. Only in $65 \%$ of these cases the outcome is vaginal birth. This implies that the corresponding sample size is 642 nulliparous women, and 1154 multiparous women who give their consent to participate in the study. Using this sample size, we achieve a suitable statistical power two-type of null hypothesis with default statistical power goals $\mathrm{p} \geq 95 \%$ and type I error $\alpha=0.05$ level of significance.
In our pilot study, a cervical dilatation of more than 4 $\mathrm{cm}$ was noted in $16.34 \%$ of the nulliparous women and $37.5 \%$ of the multiparous women who were admitted to the hospital with labour criteria. In such cases, data from the beginning of labour will not be available for calculation. In order to achieve the sample mentioned above with patients registered from the beginning of labour, we adjusted the study size to include 767 nulliparous women and 1846 multiparous women.

\section{Statistical analysis}

The statistical analyses will be performed by IBM SPSS Statistics for Windows, V.22.0. (IBM Corp).

Descriptive statistics will be produced for all study variables (mother's age, height, weight, parity, gestational age, mode and time of delivery, whether labour was spontaneous or induced, use of oxytocin or epidural anaesthesia, occiput position, PA, PD, HDA, HPD). Continuous 

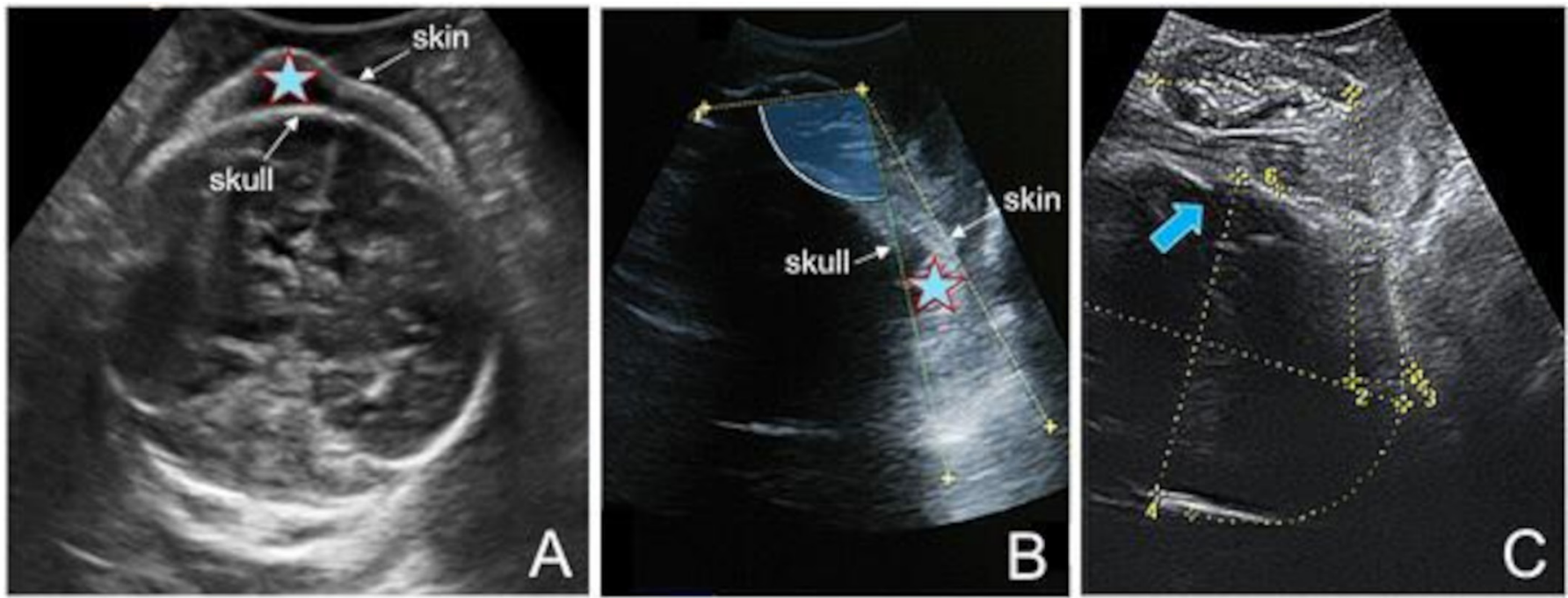

Figure $5(A, B)$ Presentation of caput (star) in transperineal transverse (A) and sagittal evaluation (B). (C) Moulding of the cranium bones indicated with the arrow.

variables will be presented as the mean and SD or median, if appropriate. Categorical data will be presented as frequency and percentage.

Data will be first tested for normality and equal variance.

Clinical obtained data from our study will be compared with similar data from other partograms. The results between groups (maternal, labour and neonatal characteristics of women assessed by classic clinical partograms or a more recent partogram and our sonopartogram) will be compared using $\mathrm{X}^{2}$ test or Fisher's exact test (for categorical variables), and Student's t-test or Mann-Whitney test where applicable (for continuous variables) with a statistical significance level set at $\mathrm{p}<0.05$.

We will analyse the agreement between sonopartogram and clinical partogram in estimating FHPo and fetal head station. For the FHPo, we will assess the level of agreement between US and digital VE using Cohen's kappa statistics. Correlation coefficient (Pearson's correlation or the Spearman's rank correlation or Kendall's rank correlation if appropriate) and linear regression will be employed for analysing the strength of association between the fetal head station estimated by digital VE and the US parameters (HPD, PA, PD and HDA).

Pearson's correlation and regressions will be used for the evaluation of correlation between US parameters (PA, PD, HDA and HPD) and between US and time to delivery and digital VE (head station) for various clinical situations (nulliparous and multiparous, fetuses with OA and those with persistent OP position).

Reference ranges $(90 \%$ range between 5th and 95th centiles) and the 95\% CI will be constructed for each US parameter, and evolution in time will be displayed in graphic form separately for nulliparous and for multiparous. Predictive ability of each US parameter for vaginal delivery will be assessed by calculating sensitivity, specificity, positive predictive value, negative predictive value and likelihood ratio and by plotting receiver operating characteristic (ROC) curve.

In order to identify factors that predict vaginal birth, for each subgroup population (nulliparous and multiparous), all analyses will use appropriate (that is, logistic or linear) regression models, with results presented as point estimates (ORs or difference in means), 95\% CIs and $p$ values. Further secondary analyses will involve planned subgroup analyses and will use multivariable regression models. In all models, predictors (like maternal age, gestational age, clinically assessed cervical dilatation, maternal BMI) will be selected for inclusion in regression. We plan to include in our model covariates such as HPD, PA, PD, HDA and OP position. Based on the probabilities predicted by the logistic models, ROC curves will be constructed and we will calculate and report the area under the curve, sensitivity and specificity rates with $95 \%$ $\mathrm{CI}$ in predicting vaginal mode of delivery.

The time from the US examination at the beginning of active phase of labour to vaginal delivery will be evaluated with Kaplan-Meier and Cox regression analysis. Data for women with caesarean section will be censored. In the Cox regression analyses, FHPo and fetal head station parameters will be tested as possible predictive factors. In additional analyses, we will adjust for maternal age, BMI, gestational age and parity as possible confounders.

\section{Reporting of adverse events}

Prenatal ultrasonography appears to be a safe investigation method, as until today there has been no study reported suggesting otherwise (statement approved by the International Society of Ultrasound in Obstetrics and Gynecology Board in September 2011 and by the World Federation of Ultrasound in Medicine and Biology Council in August 2011). ${ }^{46}$ US is routinely used in everyday clinical practice for assessment of neonates, 
including cranial and cerebral examination. However, US involves energy exposure and that requires further investigation.

Regarding the perception of labouring women about US, there have been no reports in the literature of US causing discomfort.

All adverse events reported spontaneously by patients or observed by the obstetricians will be recorded. When an adverse event occurs, the treating physician will take all necessary and appropriate measures to ensure the safety of the patient.

\section{Ethical considerations and dissemination \\ Ethics approval and consent to participate}

Ethics approval of the study protocol was obtained from the ethics committees of the universities in the three centres. The trial is approved by the University of Medicine and Pharmacy of Craiova Committee of Ethics and Academic and Scientific Deontology (No: 18/26.02.2016).

\section{Informed consent}

The US operator on duty will be responsible for explaining the procedure to the participants and for obtaining a written informed consent from all women accepting to take part in the study.

Regarding the unforeseen complications or health damage that may occur during or after labour, the management of labour and delivery is made exclusively based on the traditional clinical evaluation, by senior physicians. The US study protocol is only observational, without any obstruction for the clinical manoeuvres. The only potential sonographic intervention in the clinical assessment of labour is due to the ethical issues regarding the neonatal outcome when instrumental delivery is attempted. Thus, the attending obstetrician will be informed in case of clinical and US discordance.

On the other hand, it is made clear to all participants that US is considered safe in the third trimester and after birth, both for the mother and the baby.

\section{Compensation and insurance for harmed patients}

There will be no special financial compensation; however, any negligence on the part of the physician may be covered by the doctor's liability insurance.

\section{Author affiliations}

${ }^{1}$ Department of Obstetrics and Gynecology, University of Medicine and Pharmacy of Craiova, Craiova, Romania

${ }^{2}$ Department of Obstetrics and Gynecology, University of Athens, Athens, Greece ${ }^{3}$ Department of Obstetrics and Gynecology, Aristotle University of Thessaloniki, Thessaloniki, Greece

${ }^{4}$ Department of Computer Science, University of Craiova, Craiova, Romania ${ }^{5}$ Department of Intensive Care and Anesthesiology, University of Medicine and Pharmacy Craiova, Craiova, Romania

${ }^{6}$ Department of Public Health, University of Medicine and Pharmacy Craiova, Craiova, Romania

Acknowledgements The authors would like to thank the study sites for their support.

Contributors DGI, PA and ST conceived and designed the study and will supervise the study implementation. DGl drafted the protocol of the study. LMD, GD, MLC,
$\mathrm{RD}, \mathrm{CP}, \mathrm{LZ}, \mathrm{MN}$ and $\mathrm{DT}$ revised and refined the study protocol for an optimal implementation and provided final approval of the version to be published. MLC, SB and RS provided methodological and statistical expertise and will conduct the statistical analysis. $\mathrm{DGl}$ and $\mathrm{RD}$ drafted a $\mathrm{PhD}$ salary grant proposal for a previous approved pilot study. GD, RD, CP, LZ, RN, DR and MF will be responsible for study management, staff training and supervision, and data centralisation. DGI, PA and DT are the directors of the sites and they will provide clinical expertise and on-site management of the study. All authors critically reviewed and approved the final version of the manuscript.

Funding A funded $\mathrm{PhD}$ project provided the resources for a previous pilot study. Currently, the study has no outside funding. The project will take place as part of $\mathrm{PhD}$ and postdoctoral studies, and we will use the opportunities to apply for future research funding.

Disclaimer The potential funding sources will play no role in data collection, analysis and interpretation, in the writing of the reports, or the decision to submit the paper for publication.

Competing interests None declared.

Patient and public involvement Patients and/or the public were not involved in the design, or conduct, or reporting, or dissemination plans of this research.

Patient consent for publication Obtained.

Provenance and peer review Not commissioned; externally peer reviewed.

Open access This is an open access article distributed in accordance with the Creative Commons Attribution Non Commercial (CC BY-NC 4.0) license, which permits others to distribute, remix, adapt, build upon this work non-commercially, and license their derivative works on different terms, provided the original work is properly cited, appropriate credit is given, any changes made indicated, and the use is non-commercial. See: http://creativecommons.org/licenses/by-nc/4.0/.

\section{ORCID iDs}

Laurentiu Mihai Dira http://orcid.org/0000-0003-1766-0534

George Daskalakis http://orcid.org/0000-0001-7108-211X

Monica Laura Cara http://orcid.org/0000-0002-5471-4717

\section{REFERENCES}

1 Sherer DM, Miodovnik M, Bradley KS, et al. Intrapartum fetal head position I: comparison between transvaginal digital examination and transabdominal ultrasound assessment during the active stage of labor. Ultrasound Obstet Gynecol 2002;19:264-8.

2 Kreiser D, Schiff E, Lipitz S, et al. Determination of fetal occiput position by ultrasound during the second stage of labor. J Matern Fetal Med 2001;10:283-6.

3 Sherer DM, Miodovnik M, Bradley KS, et al. Intrapartum fetal head position II: comparison between transvaginal digital examination and transabdominal ultrasound assessment during the second stage of labor. Ultrasound Obstet Gynecol 2002;19:264-8.

4 Akmal S, Tsoi E, Kametas N, et al. Intrapartum sonography to determine fetal head position. J Matern Fetal Neonatal Med 2002;12:172-7.

5 Chou MR, Kreiser D, Taslimi MM, et al. Vaginal versus ultrasound examination of fetal occiput position during the second stage of labor. Am J Obstet Gynecol 2004;191:521-4.

6 Dupuis $\mathrm{O}$, Ruimark S, Corinne $\mathrm{D}$, et al. Fetal head position during the second stage of labor: comparison of digital vaginal examination and transabdominal ultrasonographic examination. Eur J Obstet Gynecol Reprod Biol 2005;123:193-7.

7 Oláh KS. Reversal of the decision for caesarean section in the second stage of labour on the basis of consultant vaginal assessment. J Obstet Gynaecol 2005;25:115-6.

8 Akmal S, Tsoi E, Nicolaides KH. Intrapartum sonography to determine fetal occipital position: interobserver agreement. Ultrasound Obstet Gynecol 2004;24:421-4

9 Buchmann E, Libhaber E. Interobserver agreement in intrapartum estimation of fetal head station. Int J Gynaecol Obstet 2008;101:285-9.

10 Dupuis O, Silveira R, Zentner A, et al. Birth simulator: reliability of transvaginal assessment of fetal head station as defined by the American College of obstetricians and Gynecologists classification. Am J Obstet Gynecol 2005;192:868-74.

11 Nizard J, Haberman S, Paltieli Y, et al. Determination of fetal head station and position during labor: a new technique that combines ultrasound and a position-tracking system. Am J Obstet Gynecol 2009;200:404.e1-404.e5. 
12 Molina FS, Terra R, Carrillo MP, et al. What is the most reliable ultrasound parameter for assessment of fetal head descent? Ultrasound Obstet Gynecol 2010;36:493-9.

13 Tutschek B, Torkildsen EA, Eggebø TM. Comparison between ultrasound parameters and clinical examination to assess fetal head station in labor. Ultrasound Obstet Gynecol 2013;41:425-9.

14 Rayburn WF, Siemers KH, Legino LJ, et al. Dystocia in late labor: determining fetal position by clinical and ultrasonic techniques. Am J Perinatol 1989;6:316-9.

15 Souka AP, Haritos T, Basayiannis K, et al. Intrapartum ultrasound for the examination of the fetal head position in normal and obstructed labor. J Matern Fetal Neonatal Med 2003;13:59-63.

16 Sherer DM, Abulafia O. Intrapartum assessment of fetal head engagement: comparison between transvaginal digital and transabdominal ultrasound determinations. Ultrasound Obstet Gynecol 2003;21:430-6.

17 Akmal S, Kametas N, Tsoi E, et al. Comparison of transvaginal digital examination with intrapartum sonography to determine fetal head position before instrumental delivery. Ultrasound Obstet Gynecol 2003;21:437-40

18 Wong GY, Mok YM, Wong SF. Transabdominal ultrasound assessment of the fetal head and the accuracy of vacuum cup application. Int J Gynaecol Obstet 2007;98:120-3.

19 Barbera AF, Imani F, Becker T, et al. Anatomic relationship between the pubic symphysis and ischial spines and its clinical significance in the assessment of fetal head engagement and station during labor. Ultrasound Obstet Gynecol 2009;33:320-5.

20 Ramphul M, Ooi PV, Burke G, et al. Instrumental delivery and ultrasound: a multicentre randomised controlled trial of ultrasound assessment of the fetal head position versus standard care as an approach to prevent morbidity at instrumental delivery. BJOG 2014;121:1029-38.

21 Ramphul M, Kennelly M, Murphy DJ. Establishing the accuracy and acceptability of abdominal ultrasound to define the foetal head position in the second stage of labour: a validation study. Eur $J$ Obstet Gynecol Reprod Biol 2012;164:35-9.

22 Dietz HP, Lanzarone V. Measuring engagement of the fetal head: validity and reproducibility of a new ultrasound technique. Ultrasound Obstet Gynecol 2005;25:165-8.

23 Henrich W, Dudenhausen J, Fuchs I, et al. Intrapartum translabial ultrasound (ITU): sonographic landmarks and correlation with successful vacuum extraction. Ultrasound Obstet Gynecol 2006;28:753-60

24 Iliescu DG, Adam G, Tudorache S, et al. Quantification of fetal head direction using transperineal ultrasound: an easier approach. Ultrasound Obstet Gynecol 2012;40:607-8.

25 Ghi T, Farina A, Pedrazzi A, et al. Diagnosis of station and rotation of the fetal head in the second stage of labor with intrapartum translabial ultrasound. Ultrasound Obstet Gynecol 2009;33:331-6.

26 Barbera AF, Pombar X, Perugino G, et al. A new method to assess fetal head descent in labor with transperineal ultrasound. Ultrasound Obstet Gynecol 2009;33:313-9.

27 Kalache KD, Dückelmann AM, Michaelis SAM, et al. Transperineal ultrasound imaging in prolonged second stage of labor with occipitoanterior presenting fetuses: how well does the 'angle of progression' predict the mode of delivery? Ultrasound Obstet Gynecol 2009;33:326-30.
28 Youssef A, Maroni E, Ragusa A, et al. Fetal head-symphysis distance: a simple and reliable ultrasound index of fetal head station in labor. Ultrasound Obstet Gynecol 2013;41:419-24.

29 Torkildsen EA, Salvesen K Å, Eggebø TM. Prediction of delivery mode with transperineal ultrasound in women with prolonged first stage of labor. Ultrasound Obstet Gynecol 2011;37:702-8.

30 Eggebø TM, Hassan WA, Salvesen K $\AA$, et al. Sonographic prediction of vaginal delivery in prolonged labor: a two-center study. Ultrasound Obstet Gynecol 2014;43:195-201.

31 Eggebø TM, Økland I, Heien C, et al. Can ultrasound measurements replace digitally assessed elements of the Bishop score? Acta Obstet Gynecol Scand 2009;88:325-31.

32 Iliescu D, Antsaklis A, Antsaklis P, et al. Applications of ultrasound in prelabor and labor. Donald School J Obstet Gynecol 2012;6:257-69.

33 Iliescu DG, Tudorache S, Cernea N, et al. P13.03: correlations of the sonopartogram with classic clinical partogram and key points from a pilot study. Ultrasound Obstet Gynecol 2017;50:194-5.

34 Hassan WA, Eggebø T, Ferguson M, et al. The sonopartogram: a novel method for recording progress of labor by ultrasound. Ultrasound Obstet Gynecol 2014;43:189-94.

35 Eggebø TM. Ultrasound is the future diagnostic tool in active labor. Ultrasound Obstet Gynecol 2013;41:361-3.

36 Ghi T, Maroni E, Youssef A, et al. Sonographic pattern of fetal head descent: relationship with duration of active second stage of labor and occiput position at delivery. Ultrasound Obstet Gynecol 2014;44:82-9.

37 Friedman EA. Primigravid labor; a graphicostatistical analysis. Obstet Gynecol 1955;6:567-89.

38 Friedman E. The graphic analysis of labor. Am J Obstet Gynecol 1954;68:1568-75.

39 Zhang J, Troendle JF, Yancey MK. Reassessing the labor curve in nulliparous women. Am J Obstet Gynecol 2002;187:824-8.

40 Iliescu DG, Tudorache S, Cara ML. Acceptability of Intrapartum Ultrasound Monitoring - Experience from a Romanian Longitudinal Study. Curr Health Sci J 2015;41:355-60.

41 National Institute for Health and Care Excellence. Intrapartum care for healthy women and babies. London (UK) NICE; 2017. https:// www.nice.org.uk/guidance/cg190

42 Murphy DJ, Burke G, Montgomery AA, et al. Study Protocol. IDUS - Instrumental delivery \& ultrasound. A multi-centre randomised controlled trial of ultrasound assessment of the fetal head position versus standard care as an approach to prevent morbidity at instrumental delivery. BMC Pregnancy Childbirth 2012;12:95.

43 Eggebø TM, Gjessing LK, Heien C, et al. Prediction of labor and delivery by transperineal ultrasound in pregnancies with prelabor rupture of membranes at term. Ultrasound Obstet Gynecol 2006;27:387-91.

44 Tutschek B, Braun T, Chantraine F, et al. A study of progress of labour using intrapartum translabial ultrasound, assessing head Station, direction, and angle of descent. BJOG 2011;118:62-9.

45 Altman DG. Practical statistics for medical research. Chapman and Hall, 1991.

46 , Salvesen K, Lees C, et al, Bioeffects and Safety Committee. ISUOG-WFUMB statement on the non-medical use of ultrasound, 2011. Ultrasound Obstet Gynecol 2011;38:608. 